COMMUNICATIONS IN

ANALYSIS AND GEOMETRY

Volume 6, Number 2, 317-329, 1998

\title{
Two index theorems in odd dimensions
}

\author{
DANIEL S. FREED ${ }^{1}$
}

A recent paper of Hořava and Witten [HW]-part of the current flurry of activity in string theory-contains an anomaly computation for $S^{1} /\langle\tau\rangle \times \mathbb{R}^{10}$, where $\langle\tau\rangle$ is the cyclic group of order two generated by a reflection. It was well established 10 years ago (e.g. [AS1], [F1]) that anomalies measure nontriviality in the determinant line bundle of a family of Dirac operators, and so can be computed topologically from the Atiyah-Singer index theory. The novelty in the Horava-Witten computation is a nontrivial index in odd dimensions of a type not seen in standard index theory. We abstract two general theorems which imply the Horava-Witten result. (Naturally, we replace $\mathbb{R}^{10}$ by a compact manifold $Y^{10}$ ). Theorem A is a Lefschetz formula for an orientation-reversing isometric involution on an odd dimensional manifold. The Atiyah-Bott-Segal-Singer applications of Lefschetz theory [AB1], [ASe], [AS2] all deal with orientation-preserving isometries for which there is no nontrivial Lefschetz formula in odd dimensions [AS2, Proposition 9.3]. Alternatively, we can consider [0,1] $\times Y$ in place of $S^{1} /\langle\tau\rangle \times Y$ and then the Horava-Witten anomaly computation is a boundary value problem with local boundary conditions. Theorem B generalizes this situation and is closely related to the boundary value problem in the original proof of the Atiyah-Singer index theorem [P].

Our proofs use standard techniques, except for a small trick used to prove Theorem A. For simplicity we discuss the standard complex Dirac operator; the theorems are true for any Dirac operator. Our language refers mostly to a single operator, though the results hold for families of Dirac operators as required by the anomaly problem. In this regard we remark that Theorem A only holds modulo 2 -torsion in the $K$-theory of the parameter space, whereas Theorem B holds exactly in $K$-theory. For the anomaly problem this means that Theorem A may not be adequate to detect all global anomalies. (In the general situation of Theorem A, there is probably no fixed-point formula for the exact index.)

\footnotetext{
${ }^{1}$ The author is supported by NSF grant DMS-9307446, Presidential Young Investigators award DMS-9057144, and by the O'Donnell Foundation.
} 
I thank Edward Witten for bringing this anomaly computation to my attention and for discussions.

\section{A Lefschetz formula for orientation-reversing isometries.}

Let $X$ be a compact odd dimensional spin manifold. Suppose $\tau: X \rightarrow$ $X$ is an orientation-reversing isometric involution. Assume there exists a lift $\tilde{\tau}: S_{X} \rightarrow S_{X}$ to the complex spinor bundle $S_{X}$ on $X$ such that

$$
D_{X} \tilde{\tau}=-\tilde{\tau} D_{X},
$$

where $D_{X}$ is the Dirac operator. It follows from Lemma 1.5 below that $\tilde{\tau}^{2}$ is locally constant, so dividing by a square root of that locally constant function we may assume

$$
\tilde{\tau}^{2}=1
$$

Then the \pm 1 -eigenspaces of $\tilde{\tau}$ give a splitting of the spinor fields

$$
S(X) \cong S^{+}(X) \oplus S^{-}(X),
$$

and the Dirac operator interchanges $S^{+}(X)$ and $S^{-}(X)$. Our problem is to compute

$$
\text { index }\left[D_{X}: S^{+}(X) \longrightarrow S^{-}(X)\right] \text {. }
$$

The simplest example is $X=S^{1}=\mathbb{R} / \mathbb{Z}$ with $\tau$ the reflection $x \mapsto-x$. The spinor fields may be identified with the complex functions, the Dirac operator with $i \frac{d}{d x}$, and the splitting (1.3) is the splitting into even and odd functions. Here the index is 1 . The Horava-Witten example is the product with a fixed even dimensional manifold $Y$, in which case the index is index $D_{Y}$.

The lift $\tilde{\tau}$, if it exists, is almost unique.

Lemma 1.5. Suppose $X$ is an odd dimensional spin manifold, and $\theta$ : $S_{X} \rightarrow S_{X}$ a bundle map such that $D_{X} \theta=\theta D_{X}$. Then $\theta$ is a locally constant multiple of the identity.

If $\tilde{\tau}_{1}, \tilde{\tau}_{2}$ are two lifts of $\tau$ satisfying (1.1) and (1.2), set $\theta=\tilde{\tau}_{1} \tilde{\tau}_{2}$ to conclude that $\tilde{\tau}_{1}= \pm \tilde{\tau}_{2}$ on each component of $X$. 
Proof. Fix $x \in X$ and choose a local oriented orthonormal framing $\left\{e_{i}\right\}$ near $x$. Then if $\psi$ is a spinor field with $\psi(x)=0$, an easy computation shows

$$
0=\left(D_{X} \theta-\theta D_{X}\right) \psi(x)=\left[c\left(e^{i}\right), \theta(x)\right] \nabla_{e_{i}} \psi(x),
$$

where $\left\{e^{i}\right\}$ is the dual coframing, $c(\cdot)$ is Clifford multiplication, and $\nabla$ is the Levi-Civita covariant derivative. Fix an index $i$. Choose a set of spinor fields $\left\{\psi^{(\alpha)}\right\}_{\alpha}$ so that $\psi^{(\alpha)}(x)=0$, the derivatives $\nabla_{e_{j}} \psi^{(\alpha)}(x)=0$ for $j \neq i$, and $\left\{\nabla_{e_{i}} \psi^{(\alpha)}(x)\right\}_{\alpha}$ span the fiber $\left(S_{X}\right)_{x}$. Then (1.6) implies $\left[c\left(e^{i}\right), \theta(x)\right]=0$ for all $i$, and since the spin representation is irreducible in odd dimensions, $\theta(x)$ is a scalar. Then for any spinor field $\psi$,

$$
0=\left(D_{X} \theta-\theta D_{X}\right) \psi=c(d \theta) \psi
$$

from which $d \theta=0$ so that $\theta$ is locally constant.

Concerning the existence of $\tilde{\tau}$, we recall that in odd dimensions the spin representation $\mathbf{S}$ extends to an ungraded module for the Clifford algebra on which the volume form, suitably normalized, acts as +1 . In particular, $\mathbf{S}$ is a representation of the Pin group. Now the isometry $\tau$ lifts to the bundle of orthonormal frames $O(X)$ of $X$. The spin structure induces a pin structure Pin $(X)$ - a principal Pin bundle which double covers $O(X)$-and it is a topological question about covering spaces to determine if $\tau$ acting on $O(X)$ lifts to $\operatorname{Pin}(X)$. If so, the lift may have order 4 . In any case the spinor bundle $S_{X}$ is associated to $\operatorname{Pin}(X)$, and the lift induces a map $\tilde{\tau}$ on spinor fields. But Clifford multiplication is not a map of Pin representations-there is a sign for elements which reverse the orientation - and so the Dirac operator does not extend simply extend to the Pin bundle. Rather, the sign means that the lift $\tilde{\tau}$ of an orientation-reversing isometry anticommutes with the Dirac operator as in (1.1).

We turn now to the index (1.4). The general Lefschetz formulas of Atiyah-Bott-Segal-Singer [AB1], [ASe], [AS2] apply to an elliptic operator $D: C^{\infty}(E) \rightarrow C^{\infty}(F)$ acting between two vector bundles $E, F$ with endomorphisms $\hat{\tau}_{E}, \hat{\tau}_{F}$ such that

$$
D \hat{\tau}_{E}=\hat{\tau}_{F} D .
$$

Our problem concerns the Dirac operator $D_{X}: C^{\infty}\left(S_{X}\right) \rightarrow C^{\infty}\left(S_{X}\right)$, but the given lift $\tilde{\tau}$ satisfies (1.1), not (1.7). Here is the trick: Define

$$
\hat{\tau}=\left\{\begin{aligned}
\tilde{\tau}, & \text { on the domain copy of } S_{X} ; \\
-\tilde{\tau}, & \text { on the codomain copy of } S_{X} .
\end{aligned}\right.
$$


Now $\hat{\tau}$ satisfies (1.7)! The Lefschetz number is

$$
\begin{aligned}
L\left(\hat{\tau}, D_{X}\right) & =\left.\operatorname{Trace} \hat{\tau}\right|_{\text {Ker } D_{X}}-\left.\operatorname{Trace} \hat{\tau}\right|_{\text {Coker } D_{X}}, \\
& =2 \operatorname{index}\left[D_{X}: S^{+}(X) \longrightarrow S^{-}(X)\right],
\end{aligned}
$$

twice the index we would like to compute.

The generalized Lefschetz formulas compute this index in terms of the fixed point set $\operatorname{Fix}(\tau)$ of $\tau$. In our situation each component $F$ of $\operatorname{Fix}(\tau)$ is an even dimensional manifold. The Atiyah-Segal formula [ASe, Theorem 2.12] applies in general; we first state the result with the vastly simplifying assumption that the normal bundle $N_{F}$ to each component of $\operatorname{Fix}(\tau)$ is trivial. See Remark 1.10 following the statement of Theorem A for the formula when $N_{F}$ is only assumed orientable.

Theorem A. Let $X$ be an odd dimensional spin manifold, $\tau: X \rightarrow X$ an orientation-reversing isometric involution, and $\tilde{\tau}: S_{X} \rightarrow S_{X}$ a lift to spinors which anticommutes with the Dirac operator $D_{X}$ and satisfies $\tilde{\tau}^{2}=$ 1. Then $D_{X}$ exchanges the \pm 1 -eigenspaces $S^{ \pm}(X)$ of $\tilde{\tau}$ operating on spinor fields. Assume that each component $F$ of the fixed point set $\operatorname{Fix}(\tau)$ has trivial normal bundle. The sum over these components appears in the index formula

$$
\operatorname{index}\left[D_{X}: S^{+}(X) \longrightarrow S^{-}(X)\right]=\sum_{F} \frac{\operatorname{index} D_{F}}{2^{r(F)+1}} .
$$

Here $\operatorname{codim} F=2 r(F)+1$ and $D_{F}: S^{+}(F) \rightarrow S^{-}(F)$ is the chiral Dirac operator on $F$ relative to an orientation chosen compatibly with $\tilde{\tau}$.

The orientation is explained in the proof (see (1.20)). We make several remarks before proceeding to the proof.

Remark 1.10. More generally, suppose only that each component $F$ of the fixed point set has orientable normal bundle. Then (1.9) is replaced by

$$
\text { index }\left[D_{X}: S^{+}(X) \longrightarrow S^{-}(X)\right]=\frac{1}{2} \sum_{F} \frac{\hat{\mathcal{A}}(F)}{\operatorname{ch} \Delta\left(N_{F}\right)}[F]
$$

where $\hat{\mathcal{A}}$ is the usual characteristic class associated to Dirac, $\operatorname{ch} \Delta$ is the Chern character of the spin bundle, and the orientation of $F$ is determined below. (One does not need a spin structure to define $\operatorname{ch} \Delta$.) See [AS2, §5] for a similar result. This formula only holds rationally in families. 
Remark 1.12. For the Horava-Witten example $X=S^{1} \times Y, \tau$ is reflection on the $S^{1}$ factor, and Theorem A computes

$$
\text { index }\left[D_{X}: S^{+}(X) \longrightarrow S^{-}(X)\right]=\operatorname{index} D_{Y},
$$

which agrees with [HW]. Here $Y$ is a compact even dimensional spin manifold. According to Remark 1.15 below this only holds modulo 2-torsion in families. In the next section we show that in fact this result holds exactly (see (2.11)).

Remark 1.14. For a single operator we can use the heat kernel approach to the Lefschetz formula (see $[R],[B G V]$ for example) to derive (1.9). We write

$$
\text { index }\left[D_{X}: S^{+}(X) \longrightarrow S^{-}(X)\right]=\int_{X \times X} \operatorname{Trace}\left(\tilde{\tau}(x, y) e^{-t D_{X}^{2}}(y, x)\right) d y d x,
$$

valid for any $t$, and let $t \rightarrow 0$. The integral then localizes on the fixed point set. As always in index theory, this heat kernel approach does not generalize to the integral $K$-theory index of a family of Dirac operators.

Remark 1.15. Theorem A applies to families of Dirac operators, but only gives a result in $K(Z)\left[\frac{1}{2}\right]$, where $Z$ is the parameter space. (Below we use [ASe, Theorem 2.12]. Although this theorem is stated for $K$-theory $\otimes \mathbb{C}$, in our situation the localization of the global symbol of Dirac only involves denominators which are powers of 2.)

Remark 1.16. Theorem A also applies to (families of) real Dirac operators and Dirac operators coupled to other vector bundles. The HoravaWitten example is actually for the real Dirac operator coupled to the tangent bundle. The quantity of interest is the square root of the determinant line bundle, which is computed in $K O$-theory. (See [F2, §3] for an explanation of this square root.)

Proof of Theorem A. We apply [ASe, Theorem 2.12] which asserts

$$
L\left(\hat{\tau}, D_{X}\right)=\sum_{F} \operatorname{index}\left\{\frac{\iota_{F}^{*} \sigma\left(D_{X}\right)(\hat{\tau})}{\lambda_{-1}\left(N_{F} \otimes \mathbb{C}\right)(\hat{\tau})}\right\}
$$

where $\iota_{F}: F \hookrightarrow X$ is the inclusion, $\sigma\left(D_{X}\right) \in K_{G}(T X)$ is the symbol of Dirac, and

$$
\lambda_{-1}\left(N_{F} \otimes \mathbb{C}\right)=\sum(-1)^{i} \bigwedge^{i}\left(N_{F} \otimes \mathbb{C}\right) \in K_{G}(F)
$$


Here $G=\langle\hat{\tau}\rangle$, the cyclic group generated by $\hat{\tau}$. Evaluation on $\hat{\tau}$ is the homomorphism

$$
K_{G}(F) \stackrel{\cong}{\rightrightarrows} K(F) \otimes R(G) \rightarrow K(F)
$$

which evaluates a virtual character on $\hat{\tau}$. (For the cyclic group of order two the virtual characters are real-valued.)

We work on a fixed component $F$ of codimension $2 r(F)+1=2 r+1$. Since $N_{F}$ is assumed trivial, we have an isomorphism $N_{F} \cong L^{\oplus(2 r+1)}$ in $K_{G}(F)$, where $L$ is the trivial real line bundle with $\hat{\tau}$ acting as -1 . It follows easily that

$$
\lambda_{-1}\left(N_{F} \otimes \mathbb{C}\right)(\hat{\tau})=2^{2 r+1} .
$$

Recall that the symbol $\sigma\left(D_{X}\right)$ evaluated on a cotangent vector $\theta$ is Clifford multiplication $c(\theta): S_{X} \rightarrow S_{X}$. We need to compute this for $\theta$ a cotangent vector to $F$. First, note that $F$ is orientable, since $N_{F}$ is trivial. We fix the orientations of $F$ and $N_{F}$ below. Let $N_{F}$ have the trivial spin structure. This, together with the spin structure on $X$, induces a spin structure on $F$. Then, letting $S_{F}, S_{N_{F}}$ denote the spin bundles on the tangent and normal bundles to $F$, we have

$$
\left.S_{X}\right|_{F} \cong S_{F} \otimes S_{N_{F}} \cong S_{F}^{\oplus\left(2^{r}\right)}
$$

since the normal bundle is trivial. Therefore, $\iota_{F}^{*} \sigma\left(D_{X}\right)$ is Clifford multiplication on $2^{r}$ copies of $S_{F}$.

To compute the action of $\hat{\tau}$ we fix an equivariant tubular neighborhood of $F$, which is diffeomorphic to $F \times \mathbb{R}^{2 r+1}$, and introduce a product metric. (This computation is local, so does not use the triviality of $N_{F}$.) Let $e_{i}$ be the standard orthonormal basis of $\mathbb{R}^{2 r+1}, x^{i}$ the standard coordinates on $\mathbb{R}^{2 r+1}$, and $f$ a coordinate on $F$. Then

$$
D_{X}=D_{F}+c\left(e^{i}\right) \nabla_{e_{i}} .
$$

We claim

$$
\begin{aligned}
\tau\left(f ; x^{1}, \ldots, x^{2 r+1}\right) & =\left\langle f ;-x^{1}, \ldots,-x^{2 r+1}\right\rangle \\
(\tilde{\tau} \psi)\left(f ; x^{1}, \ldots, x^{2 r+1}\right) & = \pm i^{r+1} c\left(e^{1}\right) \ldots c\left(e^{2 r+1}\right) \psi\left(f ;-x^{1}, \ldots,-x^{2 r+1}\right) \\
& =i^{m} c\left(\omega_{F}\right) \psi\left(f ;-x^{1}, \ldots,-x^{2 r+1}\right),
\end{aligned}
$$

where $\psi$ is a spinor field, $\operatorname{dim} F=2 m$, and $\omega_{F}$ is a real volume form on $F$ with $c\left(\omega_{F}\right)^{2}=(-1)^{m}$. A routine computation shows that the first expression 
for $\tilde{\tau}$ satisfies (1.1) and $\tilde{\tau}^{2}=1$, whence the remark following Lemma 1.5 implies that this is the correct expression (with one of the signs). The second expression for $\tilde{\tau}$ follows from a simple computation with Clifford algebras. It determines $\omega_{F}$ uniquely.

Now we fix the orientation on $F$ so that $\omega_{F}$ is an oriented volume form. Then $S_{F}^{ \pm}$are the $\pm\left(i^{-m}\right)$-eigenspaces of $c\left(\omega_{F}\right)$ acting on $S_{F}$, which by (1.20) are the \pm 1 -eigenspaces of $\tilde{\tau}$. Use (1.8) and (1.19) to conclude that

$$
\iota_{F}^{*} \sigma\left(D_{X}\right)(\hat{\tau})=2^{r+1} \sigma\left(D_{F}\right) .
$$

The desired result (1.9) follows from (1.17), (1.21), and (1.18).

\section{An index theorem for manifolds with boundary.}

Let $X$ be a compact odd dimensional spin manifold with boundary. The orientation on $X$ determines an orientation on $\partial X$ and so a splitting

$$
\left.S_{X}\right|_{\partial X} \cong S_{\partial X} \cong S_{\partial X}^{+} \oplus S_{\partial X}^{-}
$$

of the spin bundle on the boundary. This splitting leads to local boundary conditions $P^{ \pm}$for the Dirac operator $D_{X}$ : the domain of $\left(D_{X}, P^{ \pm}\right)$is the set of spinor fields $\psi$ on $X$ with

$$
\left(\left.\psi\right|_{\partial X}\right)^{ \pm}=0
$$

where $\phi=\phi^{+}+\phi^{-}$is the decomposition of a spinor field $\phi \in S(\partial X)$ relative to (2.1). These local boundary value problems are a key ingredient in the original proof of the Atiyah-Singer index theorem. Indeed [P, §17], [BW, $\S 21]$

$$
\operatorname{index}\left(D_{X}, P^{ \pm}\right)=0 .
$$

This is used to show that the index of the chiral Dirac operator on the boundary vanishes:

$$
\operatorname{index} D_{\partial X}=0 .
$$

Equation (2.3) is the assertion that the index is a bordism invariant.

We consider a mixture of these boundary conditions. Namely, we independently choose $P^{+}$or $P^{-}$on each component of the boundary. 
Theorem B. Let $X$ be a compact odd dimensional spin manifold with boundary, and $\partial X=\sqcup_{i} Y_{i}$ the decomposition of the boundary into components. For each $i$ choose $\epsilon_{i}=+$ or $\epsilon_{i}=-$ and consider the Dirac operator $\left(D_{X}, P^{\epsilon}\right)$ whose domain is the set of spinor fields $\psi$ such that

$$
\left(\left.\psi\right|_{Y_{i}}\right)^{\epsilon_{i}}=0
$$

Then

$$
\operatorname{index}\left(D_{X}, P^{\epsilon}\right)=\sum_{\substack{i \text { with } \\ \epsilon_{i}=-}} \operatorname{index} D_{Y_{i}}=-\sum_{\substack{i \text { with } \\ \epsilon_{i}=+}} \operatorname{index} D_{Y_{i}}
$$

Note that the last equality follows directly from (2.3). Also, if all $\epsilon_{i}=+$ or all $\epsilon_{i}=-$, then (2.5) reduces to (2.2) in view of (2.3). As is evident from the proof below, Theorem B is a direct consequence of well-known facts about boundary-value problems for Dirac operators.

Remark 2.6. Theorem B also holds in families; then (2.5) is an exact equation in $K(Z)$, where $Z$ is the parameter space. (Contrast with Theorem A which only holds in $K(Z)\left[\frac{1}{2}\right]$.) As with Theorem A (see Remark 1.16), Theorem B holds for (families of) real Dirac operators and Dirac operators coupled to other vector bundles.

Remark 2.7. Consider $X=\left[0, \frac{1}{2}\right] \times Y$, where $Y$ is a closed even dimensional spin manifold. We use the product metric. Then $\partial X=Y_{0} \sqcup Y_{\frac{1}{2}}$, where $Y_{\frac{1}{2}} \cong Y$ and $Y_{0} \cong-Y$. Here ' $-Y$ ' denotes $Y$ with the opposite orientation. Let $\epsilon_{0}=+$ and $\epsilon_{\frac{1}{2}}=-$. Then (2.5) gives

$$
\operatorname{index}\left(D_{X}, P^{\epsilon}\right)=\operatorname{index} D_{Y}
$$

Let $\tilde{D}: H^{+} \rightarrow H^{-}$be the Dirac operator in the Horava-Witten example (1.13). Here we are working on $S^{1} \times Y$ and $\psi \in H^{ \pm}=S^{ \pm}\left(S^{1} \times Y\right)$ is an $S(Y)$-valued function on $S^{1}=\mathbb{R} / \mathbb{Z}$ satisfying

$$
\psi(-x)= \pm i^{m} c\left(\omega_{Y}\right) \psi(x),
$$

where $\omega_{Y}$ is a volume form on $Y$ and $\operatorname{dim} Y=2 m$ (cf. (1.20)). We now give an a priori argument that

$$
\operatorname{index}\left(D_{X}, P^{\epsilon}\right)=\operatorname{index} \tilde{D},
$$


even in families. This is consistent with the computations (1.13) and (2.8) from Theorem A and Theorem B for single operators, and gives the exact result

$$
\text { index } \tilde{D}=\operatorname{index} D_{Y}
$$

in families. (This was previously proved modulo 2-torsion.)

To prove (2.10) note that relative to the splitting $S(Y) \cong S^{+}(Y) \oplus S^{-}(Y)$ equation (2.9) asserts that $\psi \in H^{ \pm}$satisfies

$$
\begin{aligned}
& \psi^{+}(-x)= \pm \psi^{+}(x), \\
& \psi^{-}(-x)=\mp \psi^{-}(x) .
\end{aligned}
$$

Consider the diagram

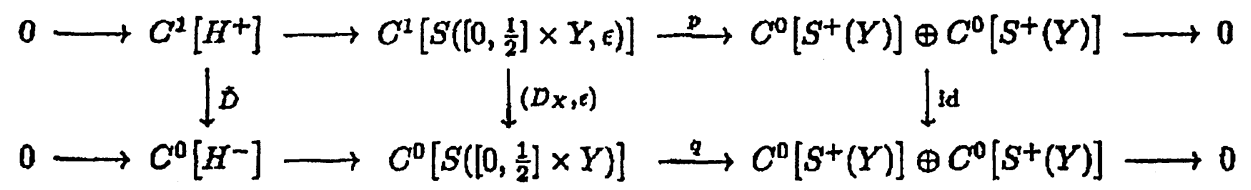

where ' $C^{1, \delta}[\cdot]$ ' and ' $C^{\delta}[\cdot]$ ' denote spaces of Hölder functions for some $0<$ $\delta<1$; ' $S\left(\left[0, \frac{1}{2}\right] \times Y, \epsilon\right)$ ' denotes the space of spinor fields satisfying (2.4), which in this case is $\psi^{-}(0)=\psi^{-}\left(\frac{1}{2}\right)=0$; the first horizontal arrows are restriction maps; and

$$
\begin{aligned}
& p(\psi)=\left\langle-i \dot{\psi}^{+}(0),-i \dot{\psi}^{+}\left(\frac{1}{2}\right)\right\rangle, \\
& q(\psi)=\left\langle\psi^{+}(0), \psi^{+}\left(\frac{1}{2}\right)\right\rangle .
\end{aligned}
$$

(Here $\dot{\psi}=\frac{d \psi}{d x}$.) A routine check shows that the rows are exact and the diagram commutes. Now (2.10) is a consequence of the following lemma. (See $[\mathrm{S}]$ for a more general discussion.)

Lemma 2.12. Let

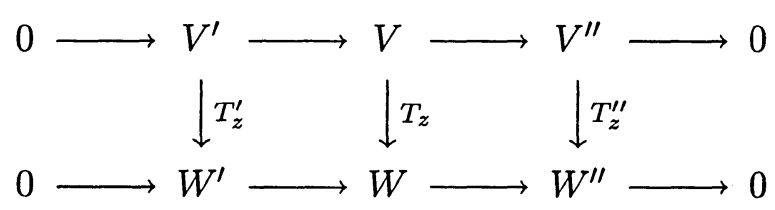


be a commutative diagram with exact rows, where $V^{\prime}, V, V^{\prime \prime}, W^{\prime}, W, W^{\prime \prime}$ are Banach spaces and $T_{z}^{\prime}, T_{z}, T_{z}^{\prime \prime}$ are Fredholm operators depending continuously on a parameter $z \in Z$. Then

$$
\operatorname{index}(T)=\operatorname{index}\left(T^{\prime}\right)+\operatorname{index}\left(T^{\prime \prime}\right) \in K(Z) .
$$

The Banach spaces are allowed to vary continuously; we omit this from the notation for convenience.

Proof. The short exact sequence of chain complexes (2.13) induces a long exact sequence in cohomology:

$$
0 \rightarrow \operatorname{Ker} T_{z}^{\prime} \rightarrow \operatorname{Ker} T_{z} \rightarrow \operatorname{Ker} T_{z}^{\prime \prime} \rightarrow \operatorname{Coker} T_{z}^{\prime} \rightarrow \operatorname{Coker} T_{z} \rightarrow \operatorname{Coker} T_{z}^{\prime \prime} \rightarrow 0 \text {. }
$$

The exactness of (2.15) proves (2.14) for a single operator. For a family it suffices to prove (2.14) for $Z$ compact. Then [AS3, §2] we can find $w_{1}^{\prime}(z), \ldots, w_{N^{\prime}}^{\prime}(z) \in W^{\prime}$ and $w_{1}(z), \ldots, w_{N}(z) \in W$ so that

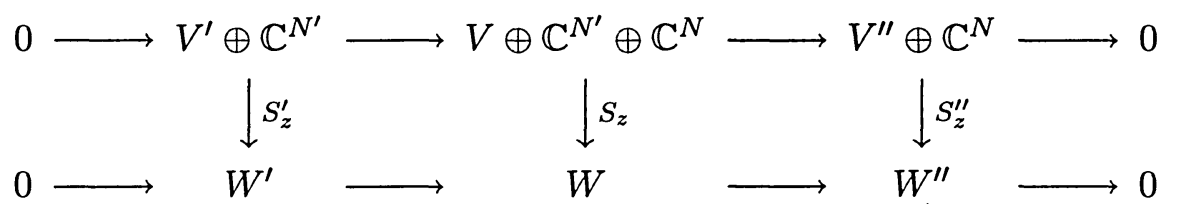

satisfies the hypotheses of the lemma and in addition $S_{z}^{\prime}, S_{z}, S_{z}^{\prime \prime}$ are surjective. Here

$$
S_{z}\left(v ; \lambda^{i} ; \mu^{j}\right)=T_{z}(v)+\lambda^{i} w_{i}^{\prime}(z)+\mu^{j} w_{j}(z)
$$

and $S_{z}^{\prime}, S_{z}^{\prime \prime}$ are the corresponding induced maps. We have

$$
\text { index } S^{\prime}=\operatorname{index} T^{\prime}+\left[Z \times \mathbb{C}^{N^{\prime}}\right] \in K(Z)
$$

with similar formulas for the indices of $S$ and $S^{\prime \prime}$. Now the exactness of (2.15) (with all cokernels vanishing) proves (2.14); the extra trivial bundles cancel out.

Proof of Theorem B. The proof is based on analysis by Calderón and Seeley $[\mathrm{P}, \S 17]$; we rely on the account in [BW]. We remark that the index with local boundary conditions is a topological invariant; in fact, it has an interpretation in $K$-theory [AB2]. So, for example, we can deform the metric to a metric which is a product near the boundary. 
Consider first a single operator. Let

$$
\hat{\mathcal{K}}=\operatorname{Ker}\left[D_{X}: S(X) \longrightarrow S(X)\right]
$$

and $\mathcal{K} \subset S(\partial X)$ the image of $\hat{\mathcal{K}}$ under restriction to the boundary. We use the Sobolev completions $H^{1}$ of $S(X)$ and $H^{1 / 2}$ of $S(\partial X)$. Then $\mathcal{K}$ is a closed infinite dimensional subspace of $S(\partial X)$. Let

$$
P^{\epsilon}: S(\partial X) \longrightarrow \bigoplus_{i} S^{\epsilon_{i}}\left(Y_{i}\right)
$$

be the projection defined by the boundary condition (2.4). The first result [BW, Theorem 20.12] is that

$$
\operatorname{index}\left(D_{X}, P^{\epsilon}\right)=\operatorname{index}\left[P_{\mathcal{K}}^{\epsilon}: \mathcal{K} \longrightarrow \bigoplus_{i} S^{\epsilon_{i}}\left(Y_{i}\right)\right]
$$

where ' $P_{\mathcal{K}}^{\epsilon}$ ' denotes the restriction of $P^{\epsilon}$ to $\mathcal{K}$. This applies in particular to $P^{+}$(which is $P^{\epsilon}$ with all $\epsilon_{i}=+$ ), and so [BW, Theorem 21.2]

$$
\begin{aligned}
\operatorname{index}\left(D_{X}, P^{\epsilon}\right) & -\operatorname{index}\left(D_{X}, P^{+}\right)=\operatorname{index}\left(P_{\mathcal{K}}^{\epsilon}\right)-\operatorname{index}\left(P_{\mathcal{K}}^{+}\right) \\
& =\operatorname{index}\left(P_{\mathcal{K}}^{\epsilon}\right)+\operatorname{index}\left(P_{\mathcal{K}}^{+}\right)^{*} \\
& =\operatorname{index}\left[P_{\mathcal{K}}^{\epsilon}\left(P_{\mathcal{K}}^{+}\right)^{*}: \bigoplus_{\substack{i \text { with } \\
\epsilon_{i}=-}} S^{+}\left(Y_{i}\right) \longrightarrow \bigoplus_{\substack{i \text { with } \\
\epsilon_{i}=-}} S^{-}\left(Y_{i}\right)\right]
\end{aligned}
$$

The final step is the assertion (see [BW, Theorem 21.5]) that $P_{\mathcal{K}}^{\epsilon}\left(P_{\mathcal{K}}^{+}\right)^{*}$ is a pseudodifferential operator of order 0 whose symbol-up to a factor and after restriction to the sphere bundle - is the symbol of the Dirac operator $\sum_{i \text { with }} D_{Y_{i}}$. (This is the brunt of the argument; it depends on properties of $\epsilon_{i}=-$

the Calderón projector.) Then the first equality in (2.5) follows directly from (2.17) and (2.2).

We briefly consider how to modify this argument for a family of Dirac operators parameterized by $z \in Z$. It suffices to consider $Z$ compact for index computations. Then as in the proof of the lemma above we can find a finite number of spinor fields $\psi_{1}(z), \ldots, \psi_{N}(z)$ so that

$$
\begin{aligned}
\left(T(z), P^{\epsilon}(z)\right): S_{P^{\epsilon}(z)}(X) \oplus \mathbb{C}^{N} & \longrightarrow S(X) \\
\left\langle\psi ; \lambda^{i}\right\rangle & \longmapsto D_{X}(z) \psi+\lambda^{i} \psi_{i}(z)
\end{aligned}
$$


is surjective. Here $S_{P^{\epsilon}(z)}(X) \subset S(X)$ is the subspace of spinor fields satisfying the boundary condition $P^{\epsilon}(z)$. Then

$$
\text { index }\left(T, P^{\epsilon}\right)=\operatorname{index}\left(D_{X}, P^{\epsilon}\right)+\left[Z \times \mathbb{C}^{N}\right] \in K(Z) .
$$

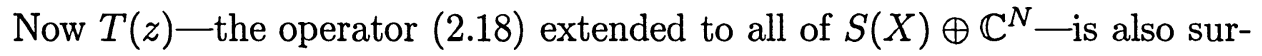
jective. Thus the orthogonal complement to the kernel of $T(z)$ varies continuously in $z$ (using $T(z)$ as an isomorphism to the continuously varying codomains), whence the kernel $\hat{\mathcal{K}}(z)$ of $T(z)$ varies continuously as well. So does its image $\mathcal{K}(z)$ in $S(\partial X)$ Equation (2.16) is replaced by

$$
\operatorname{index}\left(T, P^{\epsilon}\right)=\operatorname{index}\left[P^{\epsilon}: \mathcal{K} \longrightarrow \bigoplus_{i} S^{\epsilon_{i}}\left(Y_{i}\right)\right] .
$$

This follows simply by identifying the kernel bundle of the families of operators on each side; the cokernels vanish. By adding more $\psi_{i}(z)$ we can ensure that (2.18) is also surjective for $\left(T(z), P^{+}(z)\right)$ and repeat (2.19) and (2.20) for $P^{+}$replacing $P^{\epsilon}$. Then equation (2.17) holds-the auxiliary trivial bundle cancels out-and the proof concludes as before.

\section{References.}

[AB1] M.F. Atiyah and R. Bott, The Lefschetz fixed point theorem for elliptic complexes I, Ann. of Math. 88 (1967), 374-407; The Lefschetz fixed point theorem for elliptic complexes II, Ann. of Math. 88 (1968), 451-491.

[AB2] M.F. Atiyah and R. Bott, The index theorem for manifolds with boundary, in Differential Analysis, Proc. Bombay Symposium 1964, Oxford University Press, 175-86.

[AS1] M.F. Atiyah and I.M. Singer, Dirac operators coupled to vector potentials, Proc. Nat. Acad. Sci. 81 (1984), 2597.

[AS2] M.F. Atiyah and I.M. Singer, The index of elliptic operators, III, Ann. of Math. 87 (1968), 546-604.

[AS3] M.F. Atiyah and I.M. Singer, The index of elliptic operators, IV, Ann. Math. 93 (1971), 119-138.

[ASe] M.F. Atiyah and G. B. Segal, The index of elliptic operators, II, Ann. of Math. 87 (1968), 531-545.

[BGV] N. Berline, E. Getzler and M. Vergne, Heat Kernels and Dirac Operators, Springer-Verlag, Berlin 1992. 
[BW] B. Booss-Bavnbek and K. P. Wojciechowski, Elliptic Boundary Problems for Dirac Operators, Birkhäuser, Boston 1993.

[F1] D.S. Freed, Determinants, torsion, and strings, Commun. Math. Phys. 107 (1986), 483-513.

[F2] D.S. Freed, On determinant line bundles, in Mathematical Aspects of String Theory, ed. S. T. Yau,World Scientific Publishing 1987.

[HW] P. Hor̆ava and E. Witten, Heterotic and type I string dynamics from eleven dimensions, Nucl. Phys. B, 460 (1996), 506-524.

[P] R.S. Palais, ed., Seminar on the Atiyah-Singer index theorem, Princeton University Press, Princeton 1965, Ann. of Math. Studies 57.

[R] J. Roe, Elliptic operators, topology and asymptotic methods, Longman Scientific \& Technical, Essex, England 1988, Pitman Research Notes in Mathematics Series 179.

[S] G. Segal, Fredholm complexes, Quart. J. Math. 21 (1970), 385-402.

RECEIVED MAY 6, 1996.

UNIVERSITY OF TEXAS,

Austin, TX 78712

E-mail address: DAFR@MATH.UTEXAS.EDU 